Article

\title{
Analytical Solution of Generalized Space-Time Fractional Cable Equation
}

\section{Ram K. Saxena ${ }^{1, \dagger}$, Zivorad Tomovski ${ }^{2,3, *}$ and Trifce Sandev ${ }^{4,5, \dagger}$}

1 Department of Mathematics and Statistics, Jai Narain Vyas University, Jodhpur 342004, India; E-Mail: ram.saxena@yahoo.com

2 Department of Mathematics, University of Rijeka, Radmile Matejcic 2, 51000 Rijeka, Croatia

3 Faculty of Natural Sciences and Mathematics, Institute of Mathematics, Saints Cyril and Methodius University, 1000 Skopje, Macedonia

4 Radiation Safety Directorate, Partizanski odredi 143, P.O. Box 22, 1020 Skopje, Macedonia; E-Mail: trifce.sandev@drs.gov.mk

5 Max Planck Institute for the Physics of Complex Systems, Noethnitzer Strasse 38, 01187 Dresden, Germany

$\dagger$ These authors contributed equally to this work.

* Author to whom correspondence should be addressed; E-Mail: zivorad.tomovski@math.uniri.hr.

Academic Editor: Hari M. Srivastava

Received: 07 March 2015 / Accepted: 02 April 2015 / Published: 9 April 2015

\begin{abstract}
In this paper, we consider generalized space-time fractional cable equation in presence of external source. By using the Fourier-Laplace transform we obtain the Green function in terms of infinite series in H-functions. The fractional moments of the fundamental solution are derived and their asymptotic behavior in the short and long time limit is analyzed. Some previously obtained results are compared with those presented in this paper. By using the Bernstein characterization theorem we find the conditions under which the even moments are non-negative.
\end{abstract}

Keywords: fractional cable equation; Mittag-Leffler functions; H-function; moments 


\section{Introduction}

The cable equation has been used to model electrotonic properties of neuronal dendrities [1]. In the work of Baer and Rinzel [2], for the first time, theoretical study of wave propagation mediated by dendritic spine is performed, where the dendritic tissue is modeled with the classical cable equation. The classical cable equation which models the membrane potential $V=V(x, t)$ along the axial $x$-direction of a dendrite with diameter $d$, relative to the resting membrane potential $V_{\text {rest }}$, is given by

$$
c_{m} r_{m} \frac{d V\left(x^{*}, t^{*}\right)}{d t^{*}}=\frac{d r_{m}}{4 r_{L}} \frac{d^{2} V\left(x^{*}, t^{*}\right)}{d x^{* 2}}-\frac{V-V_{\text {rest }}}{r_{m}}+r_{m} i_{e}\left(x^{*}, t^{*}\right)
$$

where $r_{m}$ denotes the specific membrane resistance, $r_{L}$ is the longitudinal resistivity, $c_{m}$ denotes the membrane capacitance per unit area, and $i_{e}$ is the external injected current per unit area. The product $\tau=c_{m} r_{m}$ is the time constant for the dendrite.

The cable equation can be derived from the Nernst-Planck equation for electrodiffusive motion of ions [3]. There are generalizations of the cable Equation (1) where the non-local nature of the diffusive transport of living cells is taken into consideration. In the excellent works of Henry, Langlands and Wearne [4-6], time fractional cable models for spiny neuronal dendrities are introduced and investigated in detail. They derived the time fractional cable equations from fractional Nernst-Planck equations, and investigated the electrotonic effects of the trapping properties of spines [4]. In the work of Li and Deng [7], space-time fractional cable equation is introduced where the spatial non-local effects are taken into account by modifying the Ohm's law to fractional one. Thus, Li and Deng considered the following space-time fractional cable equation in the infinite domain $-\infty<x<\infty$

$$
\frac{\partial V(x, t)}{\partial t}=D_{t}^{1-\alpha}\left(\Delta^{\mu} V(x, t)\right)-\lambda^{2} D_{t}^{1-\beta}(V(x, t))+f(x, t)
$$

where $x$ and $t$ are dimensionless parameters (see relation (10) from [7]), $0<\alpha \leq 1,0<\beta \leq 1, \lambda=\sqrt{\frac{d r_{m}}{4 r_{L}}}$ is the space dendrite for the dendrite, i.e., the cable, $f(x, t)=\lambda^{2} D_{t}^{1-\beta}\left(V_{\text {rest }}+r_{m} i_{e}(x, t)\right)$ is the external source (external injected current). In Equation (2), $D_{t}^{1-v}$ is the Riemann-Liouville (R-L) fractional derivative of order $1-v[8,9]$ :

$$
D_{t}^{1-v} f(t)=\frac{1}{\Gamma(v)} \frac{d}{d t} \int_{0}^{t} \frac{f(u)}{(t-u)^{1-v}} d u, \quad 0<v<1
$$

and $\Delta^{\mu}$ is the Riesz fractional operator defined by $[9,10]$

$$
\Delta^{\mu}=-\frac{1}{2 \cos \left(\frac{\mu \pi}{2}\right)}\left(\frac{\partial^{\mu}}{\partial x^{\mu}}+\frac{\partial^{\mu}}{\partial(-x)^{\mu}}\right), \quad 1<\mu \leq 2
$$

where $\frac{\partial^{\mu}}{\partial x^{\mu}}$ and $\frac{\partial^{\mu}}{\partial(-x)^{\mu}}$ are the left and right R-L fractional derivatives of order $\mu$ defined respectively by $[8,9]$ 


$$
\begin{gathered}
\frac{\partial^{\mu}}{\partial x^{\mu}} f(x)=\frac{1}{\Gamma(n-\mu)} \frac{\partial^{n}}{\partial x^{n}} \int_{-\infty}^{x} \frac{f(u)}{(x-u)^{\mu-n+1}} d u, \quad n-1<\mu \leq n, \quad n \in N \\
\frac{\partial^{\mu}}{\partial(-x)^{\mu}} f(x)=\frac{1}{\Gamma(n-\mu)} \frac{\partial^{n}}{\partial x^{n}} \int_{-\infty}^{x} \frac{f(u)}{(u-x)^{\mu-n+1}} d u, \quad n-1<\mu \leq n, \quad n \in N
\end{gathered}
$$

Here we note that the Riesz fractional operator (4) can be represented as $-(-\Delta)^{\mu / 2}$, and that it is a generalization of the Laplacian which is obtained for $\mu=2$. It represents pseudodifferential operator with Fourier symbol $-|k|^{\mu}$, i.e., $F\left[\Delta^{\mu} f(x)\right](k)=-|k|^{\mu} F[f(x)](k)[10]$.

The boundary conditions for the cable Equation (2) are given by

$$
\lim _{x \rightarrow \pm \infty} V(x, t)=0
$$

and the initial condition by

$$
V(x, 0)=g(x)
$$

Here we note that the fractional cable equation with nonlocal boundary conditions is recently considered in [11].

Equation (2) with $\lambda=0$ and $f(x, t)=0$ is the space-time fractional diffusion equation which describes the competition between the subdiffusion and Levy flights, and which can be obtained from the continuous time random walk (CTRW) theory for broad distribution of waiting times and long-tailed distribution of jump lengths [12]. Fractional diffusion equations in presence of external force were also of interest in many recent papers [12-15]. Analytical solutions of fractional differential equations and numerical methods of fractional cable equation have been considered by many authors [16-24], to name but a few.

In our work, we further generalize Equation (2) by introducing time fractional derivative of Caputo form of order $0<\gamma \leq 1$, defined by [8,9]

$$
{ }_{C} D_{t}^{\gamma} f(t)=\frac{1}{\Gamma(n-\lambda)} \int_{0}^{t} \frac{f^{(n)}(u)}{(t-u)^{\gamma-n+1}} d u, \quad n-1<\gamma \leq n, \quad n \in N
$$

which has been used in different contexts.

The application of different forms fractional derivatives [8-10,25,26] have been found to be a useful tool for modeling systems with memory, due to the power-law memory kernel contained in the convolution integral. It is shown that the fractional derivatives appear in the diffusion equation describing anomalous diffusive processes where the mean square displacement has a power-law dependence on time [12], and that they can be used for modeling aquifer problems [13,27], non-exponential relaxation problems [28,29], etc. Furthermore, time fractional derivatives are equivalent to infinitesimal generators of generalized time fractional evolutions that arise in the transition from microscopic to macroscopic time scales [28,29]. The transition from first order time derivative to the fractional one arises in many physical problems as shown by Hilfer [25,30,31]. Contrary to the time fractional derivatives, the Riesz space fractional derivative has benn shown to represent suitable tool for 
modeling Levy flights for which the second moment diverges, and, thus, the fractional moments should be calculated in order to analyze the superdiffusive behavior of the particle $[12,14]$.

Thus, the generalization of the cable equation by using time fractional derivatives and Riesz space fractional derivative take into consideration temporal memory effect and long range spatial correlations.

This paper is organized as follows. In Section 2, we consider generalized space-time fractional cable equation with a source term. The fundamental solution (Green function) is represented in terms of infinite series in H-functions. The fractional moments of the fundamental solution are derived in Section 3, and their asymptotic behaviors are analyzed. Summary is given in Section 4.

\section{Solution of the problem}

In this paper, we consider the following generalized space-time fractional cable equation

$$
\tau_{\gamma C} D_{t}^{\gamma} V(x, t)=D_{t}^{1-\alpha}\left(\Delta^{\mu} V(x, t)\right)-\lambda^{2} D_{t}^{1-\beta}(V(x, t))+f(x, t)
$$

where $\tau_{\gamma}$ is a time parameter introduced for dimensional purposes. Without loss of generality we set $\tau_{\gamma}=1$. A few words about Equation (10) are in order. Let us apply R-L time fractional integral of order $0<\gamma \leq 1$ defined by $[8,9]$

$$
I_{t}^{\gamma} f(t)=\frac{1}{\Gamma(\gamma)} \int_{0}^{t} \frac{f(u)}{(t-u)^{1-\gamma}} d u
$$

to Equation (10). By using the relation $I_{t}^{\gamma}{ }_{C} D_{t}^{\gamma} f(t)=f(t)-f(0+)$ for $0<\gamma \leq 1[8,9]$, and then applying first time derivative, we rewrite Equation (10) in form (2), i.e.,

$$
\frac{d V(x, t)}{d t}=D_{t}^{1-\gamma} D_{t}^{1-\alpha}\left(\Delta^{\mu} V(x, t)\right)-\lambda^{2} D_{t}^{1-\gamma} D_{t}^{1-\beta}(V(x, t))+D_{t}^{1-\gamma} f(x, t)
$$

where we use the relation $\frac{d}{d t} I_{t}^{\gamma} f(t)=D_{t}^{1-\gamma} f(t)$ for $0<\gamma \leq 1[8,9]$. In order Equation (12) to be generalization of the classical cable equation we need $1<\gamma+\alpha \leq 2$ and $1<\gamma+\beta \leq 2$. From Equation (12) we see that we have fractional derivative of the source term, i.e., $D_{t}^{1-\gamma} f(x, t)=\lambda^{2} D_{t}^{1-\gamma} D_{t}^{1-\beta}\left(V_{r e s t}+r_{m} i_{e}(x, t)\right)$. This additional parameter $0<\gamma \leq 1$ may be used for better fitting experimental data.

Theorem 1. The solution of Equation (10) with boundary conditions (7) and initial condition (8) is given by

$$
V(x, t)=\int_{-\infty}^{\infty} G(x-\xi, t) g(\xi) d \xi+\int_{-\infty}^{\infty} \int_{0}^{t} G_{f}(x-\xi, t-\tau) f(\xi, \tau) d \tau d \xi
$$

where

$$
G(x, t)=\frac{1}{\mu|x| \sqrt{\pi}} \sum_{n=0}^{\infty} \frac{\left(-\lambda^{2}\right)^{n} t^{(\gamma+\beta-1) n}}{n !} H_{2,3}^{2,1}\left[\frac{|x|}{2 t^{(\alpha+\gamma-1) / \mu}} \mid \begin{array}{c}
\left(1, \frac{1}{\mu}\right),\left(1+(\gamma+\beta-1) n, \frac{\gamma+\alpha-1}{\mu}\right) \\
\left(\frac{1}{2}, \frac{1}{2}\right),\left(1+n, \frac{1}{\mu}\right),\left(1, \frac{1}{2}\right)
\end{array}\right],
$$




$$
G_{f}(x, t)=\frac{1}{\mu|x| \sqrt{\pi}} \sum_{n=0}^{\infty} \frac{\left(-\lambda^{2}\right)^{n} t^{(\gamma+\beta-1) n+\gamma-1}}{n !} H_{2,3}^{2,1}\left[\frac{|x|}{2 t^{(\alpha+\gamma-1) / \mu}} \mid \begin{array}{l}
\left(1, \frac{1}{\mu}\right),\left(\gamma+(\gamma+\beta-1) n, \frac{\gamma+\alpha-1}{\mu}\right) \\
\left(\frac{1}{2}, \frac{1}{2}\right),\left(1+n, \frac{1}{\mu}\right),\left(1, \frac{1}{2}\right)
\end{array}\right]
$$

$\operatorname{Re}(\mu)>0$, and $H_{p, q}^{m, n}\left[z \mid \begin{array}{l}\left(a_{p}, A_{p}\right) \\ \left(b_{q}, B_{q}\right)\end{array}\right]$ is the Fox H-function [32-34].

Proof. By applying Laplace transform with respect to the time $t$ to Equation (10) we obtain

$$
s^{\gamma} \hat{V}(x, s)-s^{\gamma-1} g(x)=s^{1-\alpha} \Delta^{\mu} \hat{V}(x, s)-\lambda^{2} s^{1-\beta} \hat{V}(x, s)+\hat{f}(x, s)
$$

where we use the initial condition (7) and the following formula for the Laplace transform of the Caputo fractional derivative $[8,9]$

$$
L\left[D_{t}^{\gamma} V(x, t)\right](x, s)=s^{\gamma} \hat{V}(x, s)-V(x, 0+)
$$

where $L[V(x, t)](x, s)=\hat{V}(x, s), L[f(x, t)](x, s)=\hat{f}(x, s)$, and the Laplace transform of the R-L fractional derivative defined by $[8,9]$

$$
L\left[D_{t}^{1-v} V(x, t)\right](x, s)=s^{1-v} \hat{V}(x, s)-\left[D_{t}^{v-1} V(x, t)\right]_{t=0}
$$

Here we suppose that $\left[D_{t}^{v-1} V(x, t)\right]_{t=0}=0,(v=\{\alpha, \beta\})$ (please see [4]). The Fourier transform of (16) yields

$$
s^{\gamma} \tilde{\hat{V}}(k, s)-s^{\gamma-1} \tilde{g}(k)=-s^{1-\alpha}|k|^{\mu} \tilde{\hat{V}}(k, s)-\lambda^{2} s^{1-\beta} \tilde{\hat{V}}(k, s)+\tilde{\hat{f}}(k, s)
$$

where $F[\hat{V}(x, s)](k, s)=\tilde{\hat{V}}(k, s)$ is the Fourier transform of $\hat{V}(x, s), \quad \tilde{g}(k)=F[g(x)](k)$, $\tilde{\hat{f}}(k, s)=F[\hat{f}(x, s)](k, s)$, and we use that $F\left[\Delta^{\mu} f(x)\right](k)=-|k|^{\mu} \tilde{f}(k)$ [10]. Thus, we obtain

$$
\tilde{\hat{V}}(k, s)(k, s)=\frac{s^{\gamma-1}}{s^{\gamma}+s^{1-\alpha}|k|^{\mu}+\lambda^{2} s^{1-\beta}} \tilde{g}(k)+\frac{1}{s^{\gamma}+s^{1-\alpha}|k|^{\mu}+\lambda^{2} s^{1-\beta}} \tilde{\hat{f}}(k, s) .
$$

Here

$$
\tilde{\hat{G}}(k, s)=\frac{s^{\gamma-1}}{s^{\gamma}+s^{1-\alpha}|k|^{\mu}+\lambda^{2} s^{1-\beta}}=\frac{s^{\gamma+\alpha-2}}{s^{\gamma+\alpha-1}+|k|^{\mu}+\lambda^{2} s^{\alpha-\beta}}
$$

is the Green function (solution for $g(x)=\delta(x)$ i.e., $\tilde{g}(k)=1$, and $f(x, t)=0$ ), and

$$
\tilde{\hat{G}}_{f}(k, s)=\frac{1}{s^{\gamma}+s^{1-\alpha}|k|^{\mu}+\lambda^{2} s^{1-\beta}}=\frac{s^{\alpha-1}}{s^{\gamma+\alpha-1}+|k|^{\mu}+\lambda^{2} s^{\alpha-\beta}} .
$$

Thus, the solution can be represented in a form given by (13). Expanding the right hand side of (21) and (22) in a power series in $s$ (see the approach given in $[8,9]$ ), we arrive at 


$$
\begin{aligned}
& \tilde{\hat{G}}(k, s)=\sum_{n=0}^{\infty}\left(-\lambda^{2}\right)^{n} \frac{s^{(\alpha-\beta) n+\gamma+\alpha-2}}{\left(s^{\gamma+\alpha-1}+|k|^{\mu}\right)^{n+1}}, \\
& \tilde{\hat{G}}_{f}(k, s)=\sum_{n=0}^{\infty}\left(-\lambda^{2}\right)^{n} \frac{s^{(\alpha-\beta) n+\alpha-1}}{\left(s^{\gamma+\alpha-1}+|k|^{\mu}\right)^{n+1}} .
\end{aligned}
$$

Using the Laplace transform formula for the three parameter Mittag-Leffler (M-L) function $E_{\alpha, \beta}^{\delta}(z)=\sum_{n=0}^{\infty} \frac{(\delta)_{n}}{\Gamma(\alpha n+\beta)} \frac{z^{n}}{n !}\left((\delta)_{n}=\Gamma(\delta+n) / \Gamma(\delta)\right.$ is the Pochhammer symbol) [35]:

$$
L\left[t^{\beta-1} E_{\alpha, \beta}^{\delta}\left(-a t^{\alpha}\right)\right](s)=\frac{s^{\alpha \delta-\beta}}{\left(s^{\alpha}+a\right)^{\delta}}, \quad\left(\operatorname{Re}(s)>|a|^{1 / \alpha}\right)
$$

it yields

$$
\begin{gathered}
\tilde{G}(k, t)=\sum_{n=0}^{\infty}\left(-\lambda^{2}\right)^{n} t^{(\gamma+\beta-1) n} E_{\gamma+\alpha-1,(\gamma+\beta-1) n+1}^{n+1}\left(-|k|^{\mu} t^{\gamma+\alpha-1}\right), \\
\tilde{G}_{f}(k, t)=\sum_{n=0}^{\infty}\left(-\lambda^{2}\right)^{n} t^{(\gamma+\beta-1) n+\gamma-1} E_{\gamma+\alpha-1,(\gamma+\beta-1) n+\gamma}^{n+1}\left(-|k|^{\mu} t^{\gamma+\alpha-1}\right) .
\end{gathered}
$$

The convergence of series in three parameter M-L functions of form (26) and (27) is shown in [36]. For various relations and applications of the M-L functions we refer to [37]. If we use the relation between the three parameter M-L function and the H-function [33]

$$
E_{\alpha, \beta}^{\delta}(-z)=\frac{1}{\Gamma(\delta)} H_{1,2}^{1,1}\left[z \mid \begin{array}{c}
(1-\delta, 1) \\
(0,1),(1-\beta, \alpha)
\end{array}\right]
$$

we obtain

$$
\begin{gathered}
\tilde{G}(k, t)=\sum_{n=0}^{\infty} \frac{\left(-\lambda^{2}\right)^{n}}{n !} t^{(\gamma+\beta-1) n} H_{1,2}^{1,1}\left[|k|^{\mu} t^{\gamma+\alpha-1} \mid \begin{array}{c}
(-n, 1) \\
(0,1),(-(\gamma+\beta-1) n, \gamma+\alpha-1)
\end{array}\right], \\
\tilde{G}_{f}(k, t)=\sum_{n=0}^{\infty} \frac{\left(-\lambda^{2}\right)^{n}}{n !} t^{(\gamma+\beta-1) n+\gamma-1} H_{1,2}^{1,1}\left[|k|^{\mu} t^{\gamma+\alpha-1} \mid \begin{array}{c}
(-n, 1) \\
(0,1),(1-\gamma-(\gamma+\beta-1) n, \gamma+\alpha-1)
\end{array}\right] .
\end{gathered}
$$

The inverse Fourier transform of above expressions

$$
\begin{gathered}
G(x, t)=\frac{1}{2 \pi} \sum_{n=0}^{\infty} \frac{\left(-\lambda^{2}\right)^{n}}{n !} t^{(\gamma+\beta-1) n} \int_{-\infty}^{\infty} e^{-i k x} H_{1,2}^{1,1}\left[|k|^{\mu} t^{\gamma+\alpha-1} \mid \begin{array}{c}
(-n, 1) \\
(0,1),(-(\gamma+\beta-1) n, \gamma+\alpha-1)
\end{array}\right] d k, \\
G_{f}(x, t)=\frac{1}{2 \pi} \sum_{n=0}^{\infty} \frac{\left(-\lambda^{2}\right)^{n}}{n !} t^{(\gamma+\beta-1) n+\gamma-1} \int_{-\infty}^{\infty} e^{-i k x} H_{1,2}^{1,1}\left[|k|^{\mu} t^{\gamma+\alpha-1} \mid \begin{array}{c}
(-n, 1) \\
(0,1),(1-\gamma-(\gamma+\beta-1) n, \gamma+\alpha-1)
\end{array}\right] d k,
\end{gathered}
$$

can be found by using the cosine transform of the H-function with $\rho=1$ [33] 


$$
\int_{0}^{\infty} k^{\rho-1} \cos (k x) H_{p, q}^{m, n}\left[a k^{\mu} \mid \begin{array}{c}
\left(a_{p}, A_{p}\right) \\
\left(b_{q}, B_{q}\right)
\end{array}\right] d k=\frac{2^{\rho-1} \sqrt{\pi}}{x^{\rho}} H_{p+2, q}^{m, n+1}\left[a\left(\frac{2}{x}\right)^{\mu} \mid \begin{array}{c}
\mid\left(\frac{2-\rho}{2}, \frac{\mu}{2}\right),\left(a_{p}, A_{p}\right),\left(\frac{1-\rho}{2}, \frac{\mu}{2}\right) \\
\left(b_{q}, B_{q}\right)
\end{array}\right]
$$

where $\operatorname{Re}(\rho)+\mu \min _{1 \leq j \leq m} \operatorname{Re}\left(\frac{b_{j}}{B_{j}}\right)>0, \quad \operatorname{Re}(\rho)+\mu \max _{1 \leq j \leq n} \operatorname{Re}\left(\frac{a_{j}-1}{B_{j}}\right)<1, \quad \Omega=\sum_{j=1}^{n} A_{j}-\sum_{j=n+1}^{p} A_{j}+\sum_{j=1}^{m} B_{j}-\sum_{j=m+1}^{q} B_{j}$, $\Omega>0,|\arg a|<\frac{\pi \Omega}{2}$. Therefore, we find

$$
\begin{gathered}
G(x, t)=\frac{1}{\sqrt{\pi}|x|} \sum_{n=0}^{\infty} \frac{\left(-\lambda^{2}\right)^{n}}{n !} t^{(\gamma+\beta-1) n} H_{3,2}^{1,2}\left[\left(\frac{2}{|x|}\right)^{\mu} t^{\gamma+\alpha-1} \mid \begin{array}{c}
\left(\frac{1}{2}, \frac{\mu}{2}\right),(-n, 1),\left(0, \frac{\mu}{2}\right) \\
(0,1),(-(\gamma+\beta-1) n, \gamma+\alpha-1)
\end{array}\right], \\
G_{f}(x, t)=\frac{1}{\sqrt{\pi}|x|} \sum_{n=0}^{\infty} \frac{\left(-\lambda^{2}\right)^{n}}{n !} t^{(\gamma+\beta-1) n+\gamma-1} H_{3,2}^{1,2}\left[\left(\frac{2}{|x|}\right)^{\mu} \mid \begin{array}{c}
\left(\frac{1}{2}, \frac{\mu}{2}\right),(-n, 1),\left(0, \frac{\mu}{2}\right) \\
(0,1),(1-\gamma-(\gamma+\beta-1) n, \gamma+\alpha-1)
\end{array}\right] .
\end{gathered}
$$

If we use the following property of the H-function [33]

$$
H_{p, q}^{m, n}\left[z \mid \begin{array}{l}
\left(a_{p}, A_{p}\right) \\
\left(b_{q}, B_{q}\right)
\end{array}\right]=\frac{1}{\sigma} H_{p, q}^{m, n}\left[z^{1 / \sigma} \mid \begin{array}{l}
\left(a_{p}, A_{p} / \sigma\right) \\
\left(b_{q}, B_{q} / \sigma\right)
\end{array}\right], \quad \sigma>0
$$

then (32) and (33) yield

$$
\begin{gathered}
G(x, t)=\frac{1}{\mu \sqrt{\pi}|x|} \sum_{n=0}^{\infty} \frac{\left(-\lambda^{2}\right)^{n}}{n !} t^{(\gamma+\beta-1) n} H_{3,2}^{1,2}\left[\frac{2 t^{(\gamma+\alpha-1) / \mu}}{|x|} \mid \begin{array}{c}
\left(\frac{1}{2}, \frac{1}{2}\right),\left(-n, \frac{1}{\mu}\right),\left(0, \frac{1}{2}\right) \\
\left.\left(0, \frac{1}{\mu}\right),\left(-(\gamma+\beta-1) n, \frac{\gamma+\alpha-1}{\mu}\right)\right], \\
G_{f}(x, t)=\frac{1}{\mu \sqrt{\pi}|x|} \sum_{n=0}^{\infty} \frac{\left(-\lambda^{2}\right)^{n}}{n !} t^{(\gamma+\beta-1) n+\gamma-1} H_{3,2}^{1,2}\left[\frac{2 t^{(\gamma+\alpha-1) / \mu}}{|x|} \mid\left(0, \frac{1}{\mu}\right),\left(1-\gamma-(\gamma+\beta-1) n, \frac{\gamma+\alpha-1}{\mu}\right)\right] . \\
\left(\frac{1}{2}, \frac{1}{2}\right),\left(-n, \frac{1}{\mu}\right),\left(0, \frac{1}{2}\right)
\end{array}\right] .
\end{gathered}
$$

If we further use the following transformation formula for the H-function [33]

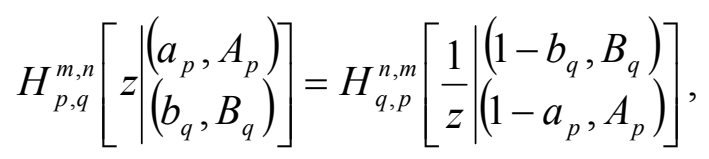

relations (35) and (36) transform into the form (14) and (15). Thus, we prove the theorem. Here we note that instead of solutions (14) and (15) one can uses the solutions (35) and (36).

Next, we consider several special cases of the fundamental solution of Equation (10).

Corollary 1. The Green function (14) in case where $\mu=2$ (second space derivative), becomes 


$$
G(x, t)=\frac{1}{2|x| \sqrt{\pi}} \sum_{n=0}^{\infty} \frac{\left(-\lambda^{2}\right)^{n} t^{(\gamma+\beta-1) n}}{n !} H_{1,2}^{2,0}\left[\frac{|x|}{2 t^{(\alpha+\gamma-1) / 2}} \mid \begin{array}{c}
\left(1+(\gamma+\beta-1) n, \frac{\gamma+\alpha-1}{2}\right) \\
\left(\frac{1}{2}, \frac{1}{2}\right),\left(1+n, \frac{1}{2}\right)
\end{array}\right] .
$$

Corollary 2. The Green function (38) in case where $\gamma=1$ is given by

$$
G(x, t)=\frac{1}{2|x| \sqrt{\pi}} \sum_{n=0}^{\infty} \frac{\left(-\lambda^{2}\right)^{n} t^{\beta n}}{n !} H_{1,2}^{2,0}\left[\frac{|x|}{2 t^{\alpha / 2}} \mid \begin{array}{c}
\left(1+\beta n, \frac{\alpha}{2}\right) \\
\left(\frac{1}{2}, \frac{1}{2}\right),\left(1+n, \frac{1}{2}\right)
\end{array}\right]
$$

which, by using the properties of $\mathrm{H}$-function [16], can be represented in a form given in [4], i.e.,

$$
G(x, t)=\frac{1}{\sqrt{4 \pi t^{\alpha}}} \sum_{n=0}^{\infty} \frac{\left(-\lambda^{2}\right)^{n} t^{\beta n}}{n !} H_{1,2}^{2,0}\left[\frac{x^{2}}{4 t^{\alpha}} \mid \begin{array}{c}
\left(1-\frac{\alpha}{2}+\beta n, \alpha\right) \\
(0,1),\left(\frac{1}{2}+n, 1\right)
\end{array}\right] .
$$

Corollary 3. The solution (40) in case of $\alpha=\beta=1$ reduces to the solution of the classical cable equation

$$
G(x, t)=\frac{1}{\sqrt{4 \pi t}} \sum_{n=0}^{\infty} \frac{\left(-\lambda^{2}\right)^{n} t^{n}}{n !} H_{0,1}^{1,0}\left[\left.\frac{x^{2}}{4 t^{\alpha}}\right|_{(0,1)} ^{-}\right]=\frac{e^{-\frac{x^{2}}{4 t^{\alpha}}}}{\sqrt{4 \pi t}} \sum_{n=0}^{\infty} \frac{\left(-\lambda^{2}\right)^{n} t^{n}}{n !}=\frac{e^{-\frac{x^{2}}{4 t}}-\lambda^{2} t}{\sqrt{4 \pi t}}
$$

as it should be. For $\lambda=0$, solution (41) turns to the Gaussian form for classical diffusion equation.

Corollary 4. The Green function (14) in case where $\gamma=1$ and $\lambda=0$ (space-time fractional diffusion equation), becomes

$$
G(x, t)=\frac{1}{\mu|x| \sqrt{\pi}} H_{2,3}^{2,1}\left[\frac{|x|}{2 t^{\alpha / \mu}}\left(\frac{1}{2}, \frac{1}{2}\right),\left(1, \frac{1}{\mu}\right),\left(1, \frac{\alpha}{\mu}\right),\left(1, \frac{1}{2}\right)\right]=\frac{1}{\mu|x|} H_{3,3}^{2,1}\left[\frac{|x|}{t^{\alpha / \mu}} \mid \begin{array}{c}
\left(1, \frac{1}{\mu}\right),\left(1, \frac{\alpha}{\mu}\right),\left(1, \frac{1}{2}\right) \\
(1,1),\left(1, \frac{1}{\mu}\right),\left(1, \frac{1}{2}\right)
\end{array}\right]
$$

which corresponds to the fundamental solution of space-time fractional diffusion equation (see for example [14,38]).

Example 1. The fundamental solution (Green function) of Equation (10), without external source term, with boundary conditions (7) and initial condition (8) is given by (14), and can be obtained if in solution (13) we set $g(x)=\delta(x)$ and $f(x, t)=0$.

If we set $\gamma=1$, we obtain the following result for the Green function given by Li and Deng [7]

$$
G(x, t)=\frac{1}{\mu|x| \sqrt{\pi}} \sum_{n=0}^{\infty} \frac{\left(-\lambda^{2}\right)^{n} t^{\beta n}}{n !} H_{2,3}^{2,1}\left[\frac{|x|}{2 t^{\alpha / \mu}} \mid \begin{array}{c}
\left(1, \frac{1}{\mu}\right),\left(1+\beta n, \frac{\alpha}{\mu}\right) \\
\left(\frac{1}{2}, \frac{1}{2}\right),\left(1+n, \frac{1}{\mu}\right),\left(1, \frac{1}{2}\right)
\end{array}\right] .
$$


Example 2. The solution (13) in case of $g(x)=0$, and external source, given by $f(x, t)=\lambda^{2}\left(V_{\text {rest }} \frac{t^{\beta-1}}{\Gamma(\beta)}+r_{m} \delta(x) \frac{t^{\beta-2}}{\Gamma(\beta-1)}\right)$, which corresponds to instantaneous input $i_{e}(x, t)=\delta(x) \delta(t)[7]$, is given by

$$
\begin{aligned}
& V(x, t)=V_{\text {rest }} \frac{\lambda^{2}}{\mu \sqrt{\pi} \Gamma(\beta)} \sum_{n=0}^{\infty} \frac{\left(-\lambda^{2}\right)^{n}}{n !} \int_{-\infty}^{\infty} \frac{1}{|x-\xi|} \int_{0}^{t} \tau^{\beta-1}(t-\tau)^{(\gamma+\beta-1) n+\gamma-1} \\
& \times H_{3,2}^{1,2}\left[\frac{2(t-\tau)^{(\gamma+\alpha-1) / \mu}}{|x-\xi|} \mid\left(\frac{1}{2}, \frac{1}{2}\right),\left(-n, \frac{1}{\mu}\right),\left(0, \frac{1}{2}\right),\left(1-\gamma-(\gamma+\beta-1) n, \frac{\gamma+\alpha-1}{\mu}\right)\right] d \tau d \xi \\
& \left.+r_{m} \frac{\lambda^{2}}{\mu \sqrt{\pi} \Gamma(\beta-1)} \sum_{n=0}^{\infty} \frac{\left(-\lambda^{2}\right)^{n}}{n !} \frac{1}{|x|} \int_{0}^{t} \tau^{\beta-2}(t-\tau)^{(\gamma+\beta-1) n+\gamma-1}\right] \\
& \times H_{3,2}^{1,2}\left[\frac{2(t-\tau)^{(\gamma+\alpha-1) / \mu}}{|x|} \mid\left(0, \frac{1}{\mu}\right),\left(1-\gamma-(\gamma+\beta-1) n, \frac{\gamma+\alpha-1}{\mu}\right)\right] d \tau .
\end{aligned}
$$

Here we note that we use solutions (35) and (36) instead of (14) and (15) in order to apply the Euler transform formula [33]

$$
\int_{0}^{t} \tau^{\rho-1}(t-\tau)^{\sigma-1} H_{p, q}^{m, n}\left[b \tau^{v} \mid \begin{array}{l}
\left(a_{p}, A_{p}\right) \\
\left(b_{q}, B_{q}\right)
\end{array}\right] d \tau=\Gamma(\sigma) t^{\rho+\sigma-1} H_{p+1, q+1}^{m, n+1}\left[\begin{array}{c}
b t^{\nu} \\
(1-\rho, v),\left(a_{p}, A_{p}\right) \\
\left(b_{q}, B_{q}\right),(1-\rho-\sigma, v)
\end{array}\right] .
$$

Therefore, for (44) we find 


$$
\begin{aligned}
& V(x, t)=V_{\text {rest }} \frac{\lambda^{2}}{\mu \sqrt{\pi}} \sum_{n=0}^{\infty} \frac{\left(-\lambda^{2}\right)^{n}}{n !} \int_{-\infty}^{\infty} \frac{1}{|\xi|} t^{(\gamma+\beta-1)(n+1)} \\
& \times H_{4,3}^{1,3}\left[\frac{2(t-\tau)^{(\gamma+\alpha-1) / \mu}}{|\xi|} \mid\left(0, \frac{1}{\mu}\right),\left(1-\gamma-(\gamma+\beta-1) n, \frac{\gamma+\alpha-1}{\mu}\right),\left(1-(\gamma+\beta-1)(n+1), \frac{\gamma+\alpha-1}{\mu}\right)\right] d \xi \\
& +r_{m} \frac{\lambda^{2}}{\mu|x| \sqrt{\pi}} \sum_{n=0}^{\infty} \frac{\left(-\lambda^{2}\right)^{n}}{n !} t^{(\gamma+\beta-1)(n+1)-1} \\
& \times H_{4,3}^{1,3}\left[\frac{2 t^{(\gamma+\alpha-1) / \mu}}{|x|} \mid \begin{array}{c}
\left(1-(\gamma+\beta-1) n-\gamma, \frac{\gamma+\alpha-1}{\mu}\right),\left(\frac{1}{2}, \frac{1}{2}\right),\left(-n, \frac{1}{\mu}\right),\left(0, \frac{1}{2}\right) \\
\left(0, \frac{1}{\mu}\right),\left(1-\gamma-(\gamma+\beta-1) n, \frac{\gamma+\alpha-1}{\mu}\right),\left(1-(\gamma+\beta-1)(n+1), \frac{\gamma+\alpha-1}{\mu}\right)
\end{array}\right] \\
& =V_{\text {rest }} \frac{\lambda^{2}}{\mu \sqrt{\pi}} \sum_{n=0}^{\infty} \frac{\left(-\lambda^{2}\right)^{n}}{n !} t^{(\gamma+\beta-1)(n+1)} \int_{-\infty}^{\infty} \frac{1}{|\xi|} \\
& \times H_{3,4}^{3,1}\left[\frac{|\xi|}{2 t^{(\gamma+\alpha-1) / \mu}} \mid \begin{array}{c}
\left(1, \frac{1}{\mu}\right),\left((\gamma+\beta-1) n+\gamma, \frac{\gamma+\alpha-1}{\mu}\right),\left((\gamma+\beta-1)(n+1), \frac{\gamma+\alpha-1}{\mu}\right) \\
\left((\gamma+\beta-1) n+\gamma, \frac{\gamma+\alpha-1}{\mu}\right),\left(\frac{1}{2}, \frac{1}{2}\right),\left(1+n, \frac{1}{\mu}\right),\left(1, \frac{1}{2}\right)
\end{array}\right] d \xi \\
& +r_{m} \frac{\lambda^{2}}{\mu|x| \sqrt{\pi}} \sum_{n=0}^{\infty} \frac{\left(-\lambda^{2}\right)^{n}}{n !} t^{(\gamma+\beta-1)(n+1)-1} \\
& \times H_{3,4}^{3,1}\left[\frac{|x|}{2 t^{(\gamma+\alpha-1) / \mu}} \mid \begin{array}{c}
\left(1, \frac{1}{\mu}\right),\left((\gamma+\beta-1) n+\gamma, \frac{\gamma+\alpha-1}{\mu}\right),\left((\gamma+\beta-1)(n+1), \frac{\gamma+\alpha-1}{\mu}\right) \\
\left((\gamma+\beta-1) n+\gamma, \frac{\gamma+\alpha-1}{\mu}\right),\left(\frac{1}{2}, \frac{1}{2}\right),\left(1+n, \frac{1}{\mu}\right),\left(1, \frac{1}{2}\right)
\end{array}\right],
\end{aligned}
$$

where we use relation (37). By using the Mellin transform of the Fox H-function

$$
\int_{0}^{\infty} x^{\xi-1} H_{p, q}^{m, n}\left[a x \mid \begin{array}{l}
\left(a_{p}, A_{p}\right) \\
\left(b_{q}, B_{q}\right)
\end{array}\right] d x=a^{-\xi} \theta(-\xi),
$$

where $\theta(s)=\frac{\prod_{j=1}^{m} \Gamma\left(b_{j}-B_{j} s\right) \prod_{j=1}^{n} \Gamma\left(1-a_{j}+A_{j} s\right)}{\prod_{j=m+1}^{q} \Gamma\left(1-b_{j}+B_{j} s\right) \prod_{j=n+1}^{p} \Gamma\left(a_{j}-A_{j} s\right)}, 0 \leq n \leq p, 1 \leq m \leq q, a_{i}, b_{j} \in C, A_{i}, B_{j} \in R^{+}, i=1,2, \ldots, p$, $j=1,2, \ldots, q$, finally we obtain

$$
\begin{aligned}
& V(x, t)=2 V_{\text {rest }} \frac{\lambda^{2}}{\mu} t^{\gamma+\beta-1} H_{1,2}^{1,1}\left[\lambda^{2} t^{\gamma+\beta-1} \mid \begin{array}{c}
(0,1) \\
(0,1),(1-(\gamma+\beta-1), \gamma+\beta-1)
\end{array}\right]+r_{m} \frac{\lambda^{2}}{\mu|x| \sqrt{\pi}} \sum_{n=0}^{\infty} \frac{\left(-\lambda^{2}\right)^{n}}{n !} t^{(\gamma+\beta-1)(n+1)-1} \\
& \times H_{3,4}^{3,1}\left[\begin{array}{c}
\left.\frac{|x|}{2 t^{(\gamma+\alpha-1) / \mu}} \mid \begin{array}{c}
\left.1, \frac{1}{\mu}\right),\left((\gamma+\beta-1) n+\gamma, \frac{\gamma+\alpha-1}{\mu}\right),\left((\gamma+\beta-1)(n+1), \frac{\gamma+\alpha-1}{\mu}\right) \\
\left((\gamma+\beta-1) n+\gamma, \frac{\gamma+\alpha-1}{\mu}\right),\left(\frac{1}{2}, \frac{1}{2}\right),\left(1+n, \frac{1}{\mu}\right),\left(1, \frac{1}{2}\right)
\end{array}\right] .
\end{array}\right.
\end{aligned}
$$


Example 3. The solution (2.4) in case of $g(x)=0$, and external source given by $f(x, t)=\lambda^{2}\left(V_{\text {rest }} \frac{t^{\beta-1}}{\Gamma(\beta)}+r_{m} \delta(x) \frac{t^{\beta-1}}{\Gamma(\beta)}\right)$, which corresponds to instantaneous input $i_{e}(x, t)=\delta(x) h(t)$ [7], where $h(t)$ is the Heaviside step function which is equal to one for $t \geq 0$ and zero otherwise, can be calculated in a same way as in Example 2. Thus, we find

$$
\begin{aligned}
& V(x, t)=2 V_{\text {rest }} \frac{\lambda^{2}}{\mu} t^{\gamma+\beta-1} H_{1,2}^{1,[}\left[\lambda^{2} t^{\gamma+\beta-1} \mid \begin{array}{c}
(0,1) \\
(0,1),(1-(\gamma+\beta-1), \gamma+\beta-1)
\end{array}\right]+r_{m} \frac{\lambda^{2}}{\mu|x| \sqrt{\pi}} \sum_{n=0}^{\infty} \frac{\left(-\lambda^{2}\right)^{n}}{n !} t^{(\gamma+\beta-1)(n+1)} \\
& \times H_{3,4}^{3,1}\left[\begin{array}{c|c|}
2 t^{(\gamma+\alpha-1) / \mu} & \left(1, \frac{1}{\mu}\right),\left((\gamma+\beta-1) n+\gamma, \frac{\gamma+\alpha-1}{\mu}\right),\left((\gamma+\beta-1)(n+1)+1, \frac{\gamma+\alpha-1}{\mu}\right) \\
\left((\gamma+\beta-1) n+\gamma-1, \frac{\gamma+\alpha-1}{\mu}\right),\left(\frac{1}{2}, \frac{1}{2}\right),\left(1+n, \frac{1}{\mu}\right),\left(1, \frac{1}{2}\right)
\end{array}\right] .
\end{aligned}
$$

\section{Fractional Moments}

Next we calculate the fractional moments [12] of the Green function (14)

$$
\left\langle|x(t)|^{\delta}\right\rangle=2 \int_{0}^{\infty} x^{\delta} G(x, t) d x, \quad \delta>0
$$

from where we analyze the anomalous diffusive behavior. For Levy flights the second moment does not exist, thus the fractional moment $\left\langle|x(t)|^{\delta}\right\rangle$ is introduced and then instead of second moment one finds $\left\langle|x(t)|^{\delta}\right\rangle^{2 / \delta}$.

Using the Mellin transform [33] for $0<\delta<\mu \leq 2$, from (47), we obtain

$$
\left\langle|x(t)|^{\delta}\right\rangle=\frac{2^{\delta+1}}{\mu \sqrt{\pi}} \sum_{n=0}^{\infty} \frac{\left(-\lambda^{2}\right)^{n} t^{(\gamma+\beta-1) n+\frac{(\gamma+\alpha-1) \delta}{\mu}}}{n !} \frac{\Gamma\left(-\frac{\delta}{\mu}\right) \Gamma\left(\frac{1}{2}+\frac{\delta}{2}\right) \Gamma\left(1+n+\frac{\delta}{\mu}\right)}{\Gamma\left(1+(\gamma+\beta-1) n+\frac{(\gamma+\alpha-1) \delta}{\mu}\right) \Gamma\left(-\frac{\delta}{2}\right)} .
$$

From (51) we see that the second moment ( $\delta=2$ ) exists only if $\mu=2$. We note that the even moments also exist only in case where $\mu=2$.

The following special cases are worth to be mentioned. For $\delta \rightarrow 0$, recalling the asymptotic formula $\frac{1}{\Gamma(z)} \sim z$ when $z<<1$ we obtain that the Green function is not normalized to one, i.e.,. it is time dependent quantity

$$
\lim _{\delta \rightarrow 0}\left\langle|x(t)|^{\delta}\right\rangle=E_{\gamma+\beta-1}\left(-\lambda^{2} t^{\gamma+\beta-1}\right)
$$

which means that the Green function is not a probability distribution function. From (52) we conclude that $\lim _{\delta \rightarrow 0}\left\langle|x|^{\delta}\right\rangle$ does not depend on parameter $\alpha$. By using the asymptotic behavior of the M-L function [39-41] for $\omega>0, \sigma>0$

$$
E_{\sigma}\left(-\omega t^{\sigma}\right) \sim \frac{t^{-\sigma}}{\omega \Gamma(1-\sigma)}, \quad t \rightarrow \infty
$$


it yields

$$
\lim _{\delta \rightarrow 0}\left\langle|x(t)|^{\delta}\right\rangle \sim \frac{t^{-(\gamma+\beta-1)}}{\lambda^{2} \Gamma(1-(\gamma+\beta-1))}, \quad t \rightarrow \infty .
$$

Since $\gamma+\beta-1>0, \int_{-\infty}^{\infty} G(x, t) d x$ shows power law decay in time.

Such non-conservation of the norm is important in certain cases, as described by the Hilfer idea of fractional generators of the dynamics [42]. The non-conservation of norm appears in the decaying of the charge density in semiconductors with exponential distribution of traps, as well as power law time decay of the ion-recombination isothermal luminescence in condensed media $[43,44]$. Here we note that non-conservation of norm has been observed in the analysis of fractional diffusion equation with composite time fractional derivative $[13,14]$.

Graphical representation of $\left\langle\mid x(t)^{0}\right\rangle$ is given in Figure 1. From the figure one can see that for classical cable equation $\gamma=\beta=1$ (solid line), $\left\langle\mid x(t)^{0}\right\rangle$ has an exponential decay to zero as it is expected from relation (52). For $\gamma=\beta=3 / 4$ (dashed line), and $\gamma=3 / 4, \beta=1 / 2$ (dot-dashed line) it shows slower power law decay to zero of form (55). Here we note that since $0<\gamma+\beta-1<1,\left\langle\mid x(t)^{0}\right\rangle$ (52) is a completely monotone function and is always positive (see for example $[45,46]$ ).

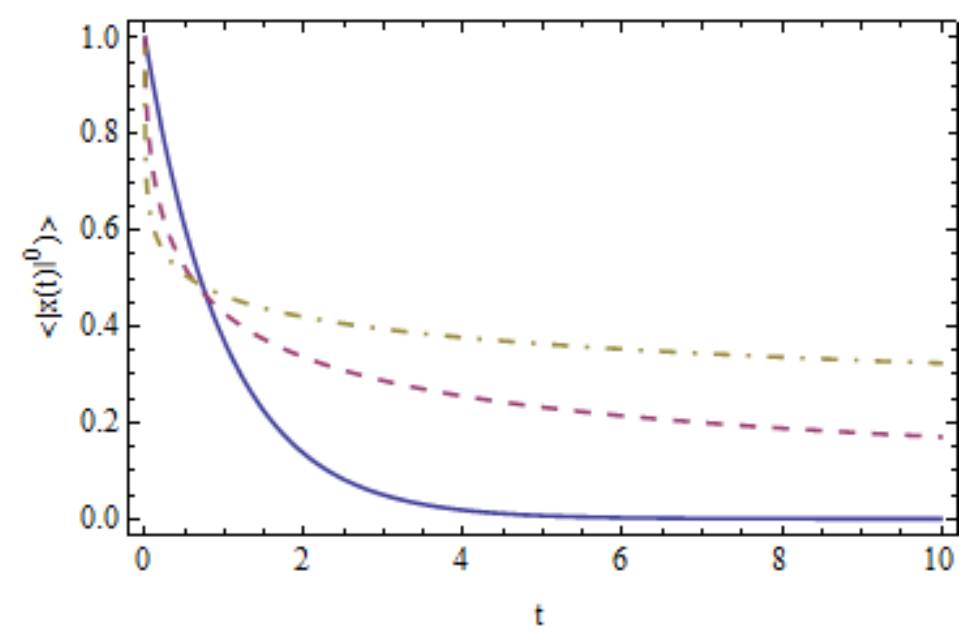

Figure 1. Graphical representation of $\left\langle|x(t)|^{0}\right\rangle$ (52) for $\lambda=1$.

For $\delta \rightarrow 2$ and $\mu \rightarrow 2$, temporal behavior of the second moment $\left\langle x^{2}(t)\right\rangle$ gives

$$
\begin{aligned}
& \lim _{\delta \rightarrow 2, \mu \rightarrow 2}\left\langle|x(t)|^{\delta}\right\rangle=\left\langle x^{2}(t)\right\rangle=2 t^{\gamma+\alpha-1} \sum_{n=0}^{\infty} \frac{(2)_{n}}{\Gamma((\gamma+\beta-1) n+\gamma+\alpha)} \frac{\left(-\lambda^{2}\right)^{n} t^{(\gamma+\beta-1) n}}{n !} \\
& =2 t^{\gamma+\alpha-1} E_{\gamma+\beta-1, \gamma+\alpha}^{2}\left(-\lambda^{2} t^{\gamma+\beta-1}\right),
\end{aligned}
$$

which in the short time limit behaves as

$$
\left\langle x^{2}(t)\right\rangle \sim \frac{2 t^{\gamma+\alpha-1}}{\Gamma(\gamma+\alpha)}
$$


and in the long time limit as

$$
\left\langle x^{2}(t)\right\rangle \sim \frac{2 t^{1+\alpha-2 \beta-\gamma}}{\lambda^{4} \Gamma(2+\alpha-2 \beta-\gamma)}
$$

where we apply the asymptotic behavior of the three parameter M-L function $[39,47,48]$.

$$
E_{\alpha, \beta}^{\delta}\left(-\omega t^{\alpha}\right) \sim \frac{t^{-\alpha \delta}}{\omega^{\delta} \Gamma(\beta-\alpha \delta)}, \quad t \rightarrow \infty .
$$

The second moment (55) gives the rate of spreading of the Green function for $\mu=2$.

Graphical representation of the second moment (55) is presented in Figure 2. Solid line corresponds to the case where $\gamma=\alpha=\beta=1$, i.e., for the classical cable equation. Power law decay of the second moment can be observed for $\gamma=3 / 4, \alpha=\beta=7 / 8$ (dashed line), which is in accordance to the behavior (57). The dot-dashed line gives the power law behavior of the second moment for $\gamma=3 / 4$, $\alpha=7 / 8, \beta=1 / 2$. The dotted line gives the behavior of the second moment for $\gamma=7 / 8, \alpha=1 / 4$, $\beta=1$. We see that it has negative sign, which seems to have no physical meaning. In order to avoid such situations we should set $2+\alpha-2 \beta-\gamma \geq 0$, which can be obtained from the analysis of the Laplace transform of the second moment (55) (see relation $(65)$, and $[45,46]$ ). However, such negativity of the second moment is interpreted in a way that for these values of the parameters the current switches directions [5]. This negativity of the second moment means that the Green function is not strictly positive for some values of parameters, i.e., it may have negative values as well.

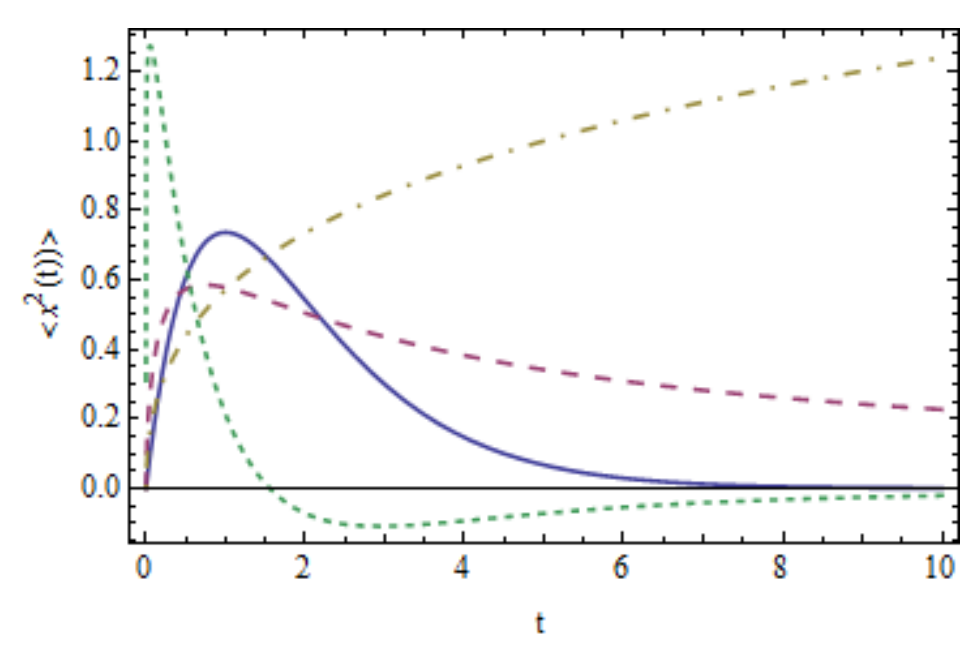

Figure 2. Graphical representation of second moment (57) for $\lambda=1$.

For $\delta \rightarrow 4$ and $\mu \rightarrow 2$, we calculate the fourth moment $\left\langle x^{4}(t)\right\rangle$ which gives

$$
\begin{aligned}
& \lim _{\delta \rightarrow 4, \mu \rightarrow 2}\left\langle|x(t)|^{\delta}\right\rangle=\left\langle x^{4}(t)\right\rangle=24 t^{2(\gamma+\alpha-1)} \sum_{n=0}^{\infty} \frac{(3)_{n}}{\Gamma((\gamma+\beta-1) n+2(\gamma+\alpha-1)+1)} \frac{\left(-\lambda^{2}\right)^{n} t^{(\gamma+\beta-1) n}}{n !} \\
& =24 t^{2(\gamma+\alpha-1)} E_{\gamma+\beta-1,2(\gamma+\alpha-1)+1}^{3}\left(-\lambda^{2} t^{\gamma+\beta-1}\right) .
\end{aligned}
$$


The fourth moment reflects the convergence of the tails of the Green function. Its calculation is important in order to derive the non-Gaussian parameter, which is defined through the ration between the fourth moment and second moment $[49,50]$. The short time limit of the fourth moment yields

$$
\left\langle x^{4}(t)\right\rangle \sim \frac{24 t^{2(\gamma+\alpha-1)}}{\Gamma(2(\gamma+\alpha-1)+1)}
$$

and the long time limit

$$
\left\langle x^{4}(t)\right\rangle \sim \frac{24 t^{1+2 \alpha-3 \beta-\gamma}}{\lambda^{6} \Gamma(2+2 \alpha-3 \beta-\gamma)} .
$$

Graphical representation of the fourth moment (59) is given in Figure 3. Same parameters as for Figure 2 are used, i.e., $\gamma=\alpha=\beta=1$ (solid line), $\gamma=3 / 4, \alpha=\beta=7 / 8$ (dashed line), $\gamma=3 / 4$, $\alpha=7 / 8, \beta=1 / 2$ (dot-dashed line). The negativity of the forth moment (dotted line, which corresponds to $\gamma=7 / 8, \alpha=1 / 4, \beta=1)$ appears since the inequality $3(\gamma+\beta-1) \leq 2(\gamma+\alpha-1)+1$ is not satisfied (see relation (65) from Remark 1).

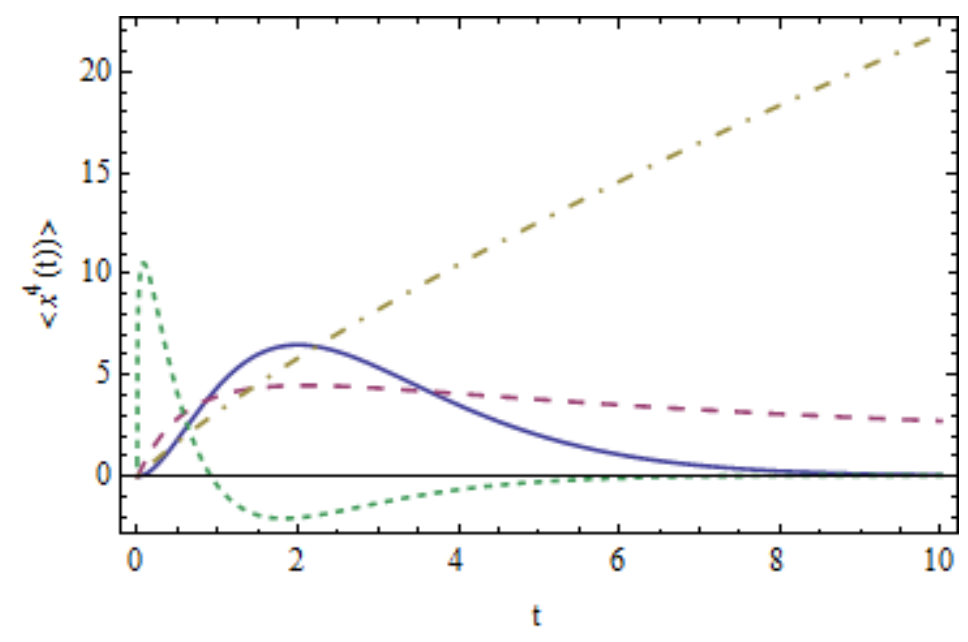

Figure 3. Graphical representation of the fourth moment (59) for $\lambda=1$.

Furthermore, we can calculate the even moments $\left\langle x^{2 m}(t)\right\rangle, m \in N$, for $\mu=2$, of the fundamental solution. They are given by three parameter M-L function in the following form

$$
\begin{aligned}
& \left\langle x^{2 m}(t)\right\rangle=\frac{2^{2 m}}{\sqrt{\pi}} \sum_{n=0}^{\infty} \frac{\left(-\lambda^{2}\right)^{n} t^{(\gamma+\beta-1) n+(\gamma+\alpha-1) m}}{n !} \\
& \times \frac{\Gamma\left(\frac{1}{2}+m\right) \Gamma(1+m+n)}{\Gamma((\gamma+\beta-1) n+(\gamma+\alpha-1) m+1)} .=(2 m) ! t^{(\gamma+\alpha-1) m} E_{\gamma+\beta-1, m(\gamma+\alpha-1)+1}^{m+1}\left(-\lambda^{2} t^{\gamma+\beta-1}\right) .
\end{aligned}
$$

From general result (62) directly follow relations (52), (55) and (59) for $m=0, m=1$ and $m=2$, respectively. 


\section{Positivity of the Even Moments}

Here we note that one can find the constraints of parameters under which the even moments (62) are non-negative. For this reason, we consider the following function

$$
K(t)=t^{(\gamma+\alpha-1) m} E_{\gamma+\beta-1, m(\gamma+\alpha-1)+1}^{m+1}\left(-\lambda^{2} t^{\gamma+\beta-1}\right) .
$$

In order (63) to be non-negative, from the Bernstein characterization theorem (see for example $[45,46,51])$, its Laplace transform

$$
f(s)=L[K(t)](s)=\frac{s^{(\gamma+\beta-1)(m+1)-(\gamma+\alpha-1) m-1}}{\left(s^{\gamma+\beta-1}+\lambda^{2}\right)^{m+1}}
$$

should be completely monotone function (given function $f:(0, \infty) \rightarrow R$ is a completely monotone if $(-1)^{n} f^{(n)}(s) \geq 0$ for all $n \in N \cup\{0\}$ and $s>0$, see Definition 1.3 on page 2 from [45]). Since $0<\gamma+\beta-1 \leq 1$ then, from Definition 3.1 on page 15 from [45], $s^{\gamma+\beta-1}$ is a Bernstein function $\left(f:(0, \infty) \rightarrow R\right.$ is a Bernstein function if $f(s) \geq 0$ for all $s>0$, and $(-1)^{n-1} f^{(n)}(s) \geq 0$ for all $n \in N$ and $s>0$ ), and thus $s^{\gamma+\beta-1}+\lambda^{2}$ is a Bernstein function too, as a sum of two Bernstein functions (see Corollary 3.7 on page 20 from [45]). From here we conclude that $\frac{1}{s^{\gamma+\beta-1}+\lambda^{2}}$ is a completely monotone function (Exercise 9.9 from [52], see also [53]), and $\frac{1}{\left(s^{\gamma+\beta-1}+\lambda^{2}\right)^{m+1}}$ is a completely monotone as a product of $(m+1)$ completely monotone functions (see Corollary 6 on page 5 from [45]). Therefore, $s^{(\gamma+\beta-1)(m+1)-(\gamma+\alpha-1) m-1}$ should be completely monotone. Function of form $s^{v}, s>0$, is completely monotone for any $v \leq 0$ [45]. From here, we conclude that the following inequality

$$
2+\alpha m-\beta(m+1)-\gamma \geq 0
$$

should be satisfied in order even moments (62) to be non-negative. The case with $\gamma=1$ yields $1+\alpha m-\beta(m+1) \geq 0$. For the classical cable equation $\alpha=\beta=\gamma=1$ all the even moments are nonnegative since (65) is satisfied for any $m \in N$. This can also be concluded from the Green function (41), which is non-negative function.

\section{Conclusions}

In this paper, a generalization of the space-time fractional cable equation is discussed. The new mathematical model takes into account the temporal memory effects and spatial non-locality. Further we derive the Green function of the generalized space-time fractional cable equation by the application of Laplace and Fourier transforms. The Green function of the proposed equation is obtained in an exact form in terms of infinite series in $\mathrm{H}$-functions. We analyze the anomalous behavior of the derived model by calculating the fractional moments of the Green function. It is shown that the Green function is not normalized, i.e., it is a time dependent quantity which shows power law decay. Such non-conservation of norm has been observed in different fractional models. We analyze the even moments and we show that they may have negative sign, which means that the Green function is not always positive, and that 
the current switches directions. By using of Bernstein characterization theorem we show under which conditions of parameters the even moments are non-negative.

\section{Acknowledgments}

The author Zivorad Tomovski is supported under the European Commission and the Croatian Ministry of Science, Education and Sports Co-Financing Agreement No. 291823. In particular, ZT acknowledges project financing from the Maria Curie FP7-PEOPLE-2011-COFUND program NEWFELPRO Grant Agreement No. 37 - Anomalous diffusion.

\section{Author Contributions}

All authors have contributed equally.

\section{Conflicts of Interest}

The authors declare no conflict of interest.

\section{References}

1. Segev, I.; London, M. Untangling dendrites with quantitative models. Science 2000, 290, 744-750.

2. Baer, S.M.; Rinzel, J. Propagation of dendritic spikes mediated by excitable spines: A continuum theory. J. Neurophysiol. 1991, 65, 874-890.

3. Qian, N.; Sejnowski, T.J. An electro-diffusion model for computing membrane potentials and ionic concentrations in branching dendrites, spines and axons. Biol. Cybern. 1989, 62, 1-15.

4. Henry, B.I.; Langlands, T.A.M.; Wearne, S.L. Fractional cable models for spiny neuronal dendrites. Phys. Rev. Lett. 2008, 100, 128103.

5. Henry, B.I.; Wearne, S.L. Fractional cable equation models for anomalous electrodiffusion in nerve cells: infinite domain solutions. J. Math. Biol. 2009, 59, 761-808.

6. Langlands, T.A.M.; Henry, B.I.; Wearne, S.L. Fractional cable equation models for anomalous electrodiffusion in nerve cells: Finite domain solutions. SIAM J. Appl. Math. 2011, 71, 1168-1203.

7. Li, C.; Deng, W. Analytical solutions, moments, and their asymptotic behaviors for the time-space fractional cable equation. Commun. Theor. Phys. 2014, 62, 54-60.

8. Podlubny, I. Fractional Differential Equations; Academin Press: San Diego, CA, USA, 1999.

9. Kilbas, A.A.; Srivastava, H.M.; Trujillo, J.J. Theory and Applications of Fractional Differential Equations; Elsevier: Amsterdam, the Netherlands, 2006.

10. Samko, S.G.; Kilbas, A.A.; Marichev, O.I. Fractional Integrals and Derivatives, Theory and Applications; Gordon and Breach: London, UK, 1993.

11. Bazhlekova, E.G.; Dimovski, I.H. Exact solution for the fractional cable equation with nonlocal boundary conditions. Cent. Eur. J. Phys. 2013, 11, 1304-1313.

12. Metzler, R.; Klafter, J. The random walk's guide to anomalous diffusion: A fractional dynamics approach. Phys. Rep. 2000, 339, 1-77.

13. Sandev, T.; Metzler, R.; Tomovski, Z. Fractional diffusion equation with a generalized Riemann-Liouville time fractional derivative. J. Phys. A: Math. Theor. 2011, 44, 255203. 
14. Tomovski, Z.; Sandev, T.; Metzler, R.; Dubbeldam, J. Generalized space-time fractional diffusion equation with composite fractional time derivative. Phys. A 2012, 391, 2527-2542.

15. Lenzi, E.K.; Rossato, R.; Lenzi, M.K.; da Silva, L.R.; Goncalves, G. Fractional diffusion equation and external forces: solutions in a confined region. Z. Naturforschung Sect. A 2010, 65, 423-430.

16. Huang, F.; Liu, F. The space-time fractional diffusion equation with Caputo derivatives. J. Appl. Math. Comput.2005, 19, 179-190.

17. Huang, F.; Liu, F. The time fractional diffusion equation and advection-dispersion equation. ANZIAM J. 2005, 46, 317-330.

18. Huang, F.; Liu, F. The fundamental solution of the space-time fractional advection-dispersion equation. J. Appl. Math. Comput. 2005, 18, 339-350.

19. Liu, F.; Anh, V.; Turner, I. Numerical solution of the space fractional Fokker-Planck equation. J. Comput. Appl. Math. 2004, 166, 209-219.

20. Liu, F.; Zhuang, P.; Anh, V.; Turner, I.; Burrage, K. Stability and Convergence of the difference Methods for the space-time fractional advection-diffusion equation. Appl. Math. Comput. 2007, 191, 12-20.

21. Liu, F.; Yang, Q.; Turner, I. Two new implicit numerical methods for the fractional cable equation. J. Comput. Nonlinear Dyn. 2010, 6, 01109.

22. Chen, C.; Liu, F.; Burrage, K. Numerical analysis for a variable-order nonlinear cable equation. J. Comput. Appl. Math. 2011, 236, 209-224.

23. Zheng, M.; Liu, F.; Turner, I.; Anh, V. A novel high order space-time spectral method for the time-fractional Fokker-Planck equation. SIAM J. Sci. Comput. 2015, 37, A701-A724.

24. Liu, F.; Chen, S.; Turner, I.; Burrage, K.; Anh, V. Numerical simulation for two-dimensional Riesz space fractional diffusion equations with a nonlinear reaction term. Cent. Eur. J. Phys. 2013, 11, 1221-1232.

25. Hilfer, R. Application of Fractional Calculus in Physics; World Scientific: Singapore, Singapore, 2000.

26. Atangana, A.; Secer, A. A note on fractional order derivative and table of fractional derivative of some special function. Abstr. Appl. Anal. 2013, 2013, 279681.

27. Atangana, A. Drawdown in prolate spheroidal-spherical coordinates obtained via Green's function and perturbation methods. Comun. Nonlin. Sci. Numer. Simul. 2014, 29, 1259-1269.

28. Hilfer, R. Experimental evidence for fractional time evolution in glass forming materials. Chem. Phys. 2002, 284, 399-408.

29. Hilfer, R. On fractional relaxation. Fractals 2003, 11, 251-257.

30. Hilfer, R. Exact solutions for a class of fractal time random walks. Fractals 1995, 3, 211-216.

31. Hilfer, R. An extension of the dynamical foundations for the statistical equilibrium concept. Phys. $A$ 1995, 221, 89-96.

32. Mathai, A.M.; Saxena, R.K. Distribution of a product and the structural set up of densities. Ann. Math. Statist. 1969, 40, 1439-1448.

33. Mathai, A.M.; Saxena, R.K.; Haubold, H.J. The H-Function: Theory and Applications; Springer: New York, NY, USA, 2010.

34. Srivastava, H.M.; Gupta, K.C.; Goyal, S.P. The H-Functions of One and Two Variables with Applications; South Asian Publishers: New Delhi, Madras, India, 1982. 
35. Prabhakar, T.R. A singular integral equation with a generalized Mittag Leffler function in the kernel. Yokohama Math. J. 1971, 19, 7-15.

36. Sandev, T.; Tomovski, Z.; Dubbeldam, J.L.A. Generalized Langevin equation with a three parameter Mittag-Leffler noise. Phy. A 2011, 390, 3627-3636.

37. Srivastava H.M.; Tomovski, Z. Fractional calculus with an integral operator containing a generalized Mittag-Leffler function in the kernel. Appl. Math. Comput. 2009, 211, 198-210.

38. Haubold, H.J.; Mathai, A.M.; Saxena, R.K. Solutions of fractional reaction-diffusion equations in terms of the H-function. Bull. Astr. Soc. India 2007, 35, 681-689.

39. Saxena, R.K.; Mathai, A.M.; Haubold, H.J. Unified fractional kinetic equation and a fractional diffusion equation. Astrophys. Space Sci. Trans. 2004, 209, 299-310.

40. Mainardi, F. Fractional Calculus and Waves in Linear Viscoelesticity: An Introduction to Mathematical Models; Imperial College Press: London, UK, 2010.

41. Seybold, H.; Hilfer, R. Numerical algorithm for calculating the generalized Mittag-Leffler function. SIAM J. Numer. Anal. 2008, 47, 69-88.

42. Hilfer, R. Classification theory for anequilibrium phase transitions. Phys. Rev. E 1993, 48, 2466.

43. Bisquert, J. Fractional diffusion in the multiple-trapping regime and revision of the equivalence with the continuous-time random walk. Phys. Rev. Let. 2003, 91, 010602.

44. Orenstein, J.; Kastner, M. Photocurrent transient spectroscopy: measurement of the density of localized states in $a$-As2 $\mathrm{Se}_{3}$. Phys. Rev. Lett. 1981, 46, 1421.

45. Schilling, R.; Song, R.; Vondracek, Z. Bernstein Functions; De Gruyter: Berlin, Germany, 2010.

46. Tomovski, Z.; Pogany, T.; Srivastava, H.M. Laplace type integral expressions for a certain three-parameter family of generalized Mittag-Leffler functions with applications involving complete monotonicity. J. Frankl. Inst. 2014, 351, 5437-5454.

47. Sandev, T.; Tomovski, Z. Langevin equation for a free particle driven by power law type noises. Phys. Lett. A 2014, 378, 1-9.

48. Sandev, T; Metzler, R.; Tomovski, Z. Correlation functions for the fractional generalized Langevin equation in the presence of internal and external noise. J. Math. Phys. 2014, 55, 023301.

49. Spanner, M.; Hoefling, F.; Schroeder-Turk, G.; Mecke, K.; Franosch, T. Anomalous transport of a tracer on percolation clusters. J. Phys.: Condens. Matter 2011, 23, 234120.

50. Metzler, R.; Jeon, J.-H.; Cherstvy, A.G.; Barkai, E. Anomalous diffusion models and their properties: non-stationarity, non-ergodicity, and aging at the centenary of single particle tracking. Phys. Chem. Chem. Phys. 2014, 16, 24128-24164.

51. Garra, R.; Gorenflo, R.; Polito, F.; Tomovski, Z. Hilfer-Prabhakar derivatives and some applications. Appl. Math. Comput. 2014, 242, 576-589.

52. Berg, C.; Forst, G. Potential Theory on Locally Compact Abelian Groups; Springer: Berlin, Germany, 1975.

53. Fujita, Y. A generalization of the results of Pillai. Ann. Inst. Statist. Math. 1993, 45, 361-365.

(C) 2015 by the authors; licensee MDPI, Basel, Switzerland. This article is an open access article distributed under the terms and conditions of the Creative Commons Attribution license (http://creativecommons.org/licenses/by/4.0/). 